\title{
Interaction of Vitamin $\mathbf{E}$ and Its Model Compounds with Unsaturated Fatty Acids in Homogeneous Solution*
}

\author{
Shiro URANo, ${ }^{* *}$ Noriko Shichita, and Mitsuyoshi Matsuo ${ }^{1}$ \\ Tokyo Metropolitan Institute of Gerontology, \\ Sakae-cho, Itabashi-ku, Tokyo 173, Japan
}

(Received October 12, 1987)

\begin{abstract}
Summary Either $\alpha$-tocopherol (vitamin E) or one of its model compounds having side chains of different length at the 2-position of $\alpha$-tocopherol, forms complexes with an unsaturated fatty acid in methanol. For complex formation, the isoprenoid side chain and hydroxy group of $\alpha$ tocopherol are unessential and, rather, the methyl groups attached to the aromatic ring of the chromanol moiety seems to be responsible. For better interaction, more than three methylene-interrupted $\mathrm{Z}$ double bonds of a fatty acid are necessary. These findings are incompatible with the hypothesis of Diplock and Lucy(l) on the interaction of vitamin E with each polyunsaturated fatty acid.
\end{abstract}

Key Words vitamin E, $\alpha$-tocopherol, complex formation, vitamin E-fatty acid interaction, fluorescence quenching, ${ }^{13} \mathrm{C}-\mathrm{NMR}$

It has been generally accepted that vitamin E, especially $\alpha$-tocopherol, acts as a biological antioxidant in the lipid core of biomembranes in which it is mostly located (2). Such biological activity is presumed to arise from the chemical reactivity of the chromanol moiety of $\alpha$-tocopherol. On the other hand, the previous investigations of biomembranes and model phospholipid bilayers have shown that $\alpha$-tocopherol decreases the permeability of these systems and enhances the stability of liposome membrane (3). Diplock and Lucy proposed that these properties result from structural interactions between the isoprenoid side chain of $\alpha$-tocopherol and the polyunsaturated fatty acid-particularly arachidonic acid-residues of membrane phospholipids (1); i.e., the physicochemical interaction of $4^{\prime} \mathrm{a}-$ and $8^{\prime} \mathrm{a}-$ methyl groups on the isoprenoid side chain with the Z-pockets of the arachidoyl chains of membrane phospholipids offers a possible explanation for the membrane-

${ }^{1}$ 浦野四郎, 七田紀子, 松尾光芳

* Issue as TMIG-I No. 109.

** To whom reprint request should be addressed. 
stabilizing property of vitamin E. No evidence, however, has been reported to show that this interaction exists in biomembranes, although the effect of $\alpha$-tocopherol on biomembranes has been studied using a variety of techniques, such as ultraviolet absorption (4), lipid monolayers $(5,6)$ and bilayers $(3,7)$, electron spin resonance $(8)$ and nuclear magnetic resonance $(8-10)$. Recently we reported that although $\alpha$ tocopherol has an affinity for the unsaturated fatty acid moieties in liposomes, 4 'aand 8'a-methyl groups on the isoprenoid side chain do not interact specifically with the arachidoyl residues of lecithin(11). Hence, our finding was inconsistent with the above hypothesis.

In order to examine details of such interaction, the complex formation of $\alpha$ tocopherol and its model compounds with fatty acids has been investigated in homogeneous solution (methanol) using fluorescence quenching and ${ }^{13} \mathrm{C}-\mathrm{NMR}$ relaxation $\left(T_{1}\right)$ techniques.

\section{MATERIALS AND METHODS}

Chemicals. Fatty acids were purchased from Sigma Chemical Co. (St. Louis, Mo, USA) and were used without purification. $d l-\alpha$-Tocopherol was obtained from E. Merck (Darmstadt, BRD) and purified by silica gel column chromatography using a mixture of $n$-hexane and ether $(10: 1)$ as an eluent. Model compounds were prepared according to the method in the previous report (12). Deuterated methanol $\left(C D_{3} \mathrm{OD}\right)$ was purchased from E. Merck. Silica gel C-200 for column chromatography was obtained from Wako Chemical Industries (Osaka, Japan) and all other chemicals from common laboratory suppliers.

Measurement of fluorescence quenching. Either $\alpha$-tocopherol or one of its model compounds at the concentration of $1.0 \times 10^{-4} \mathrm{M}$ was dissolved in methanol solution containing stearic $(18: 0)$, oleic $(18: 1)$, linoleic $(18: 2)$, linolenic $(18: 3)$, or arachidonic (20:4) acid (in a given amount), respectively. Using a Shimadzu spectrofluorophotometer RF-503A (Shimadzu Corp., Kyoto, Japan), the intensity of fluorescence was measured at $295 \mathrm{~nm}$ (ex.) and $330 \mathrm{~nm}$ (em.).

Measurement of ${ }^{13} \mathrm{C}$ spin-lattice relaxation time $\left(T_{1}\right)$. Stearic, oleic, linoleic, linolenic, and arachidonic acid in a given amount, were respectively added to $1.2 \times 10^{-3} \mathrm{M} \alpha$-tocopherol in methanol- $d_{4}$. At $20^{\circ} \mathrm{C}$, the ${ }^{13} \mathrm{C} \mathrm{T}_{1}$ relaxation times were measured on a Varian XL-200 spectrometer (Varian, Palo Alto, Calif., USA) using the inversion-reccvery technique which employs a $\left(180^{\circ}-t-90^{\circ}\right)$ pulse sequence where $t$ is the delay time between $180^{\circ}$ and $90^{\circ}$ pulses.

\section{RESULTS AND DISCUSSION}

As can be seen in Fig. 2, when a fatty acid was mixed in 'methanol at a specified molar ratio with either $\alpha$-tocopherol or one of its model compounds (Fig. 1, hereinafter referred to collectively by the term of "chromanol"), intensity of fluorescence at $295 \mathrm{~nm}$ (ex.) and $330 \mathrm{~nm}$ (em.) decreased with an increase in the 


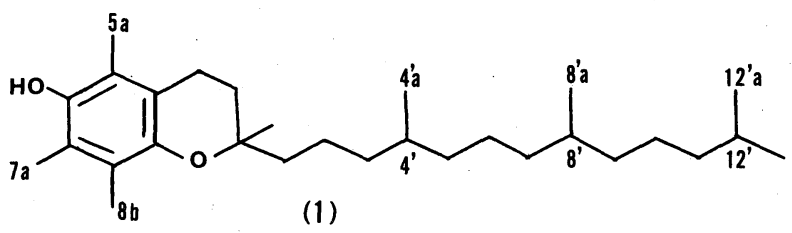<smiles>CCC(C)CCCC1(C)CCc2c(C)c(O)c(C)c(C)c2O1</smiles>

Fig. 1. Structures of $\alpha$-tocopherol and the model compounds. (1), $n=3$ ( $\alpha$-tocopherol); (2), $n=2 ;(3), n=1 ;(4), n=0$.

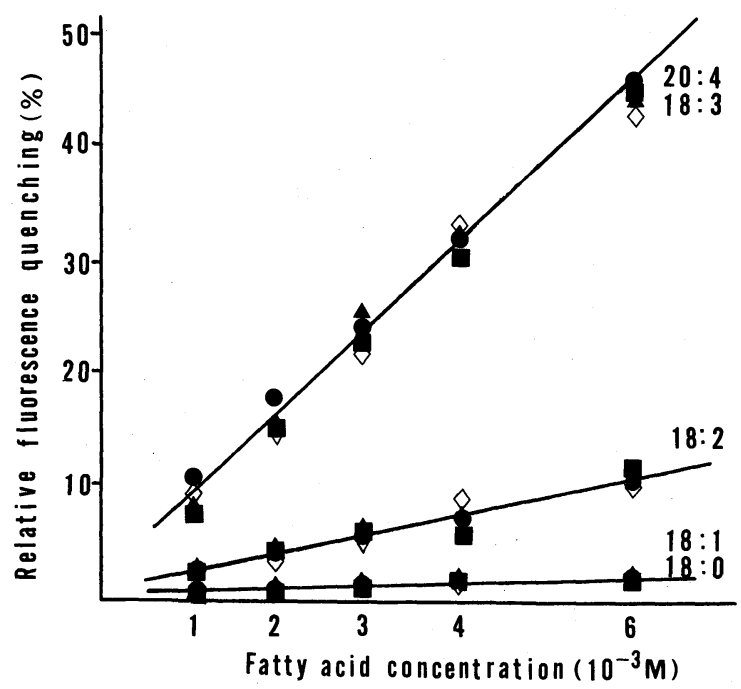

Fig. 2. Relative fluorescence quenching of $\alpha$-tocopherol and its model compounds in methanol by fatty acid concentration. Stearic $(18: 1)$, oleic $(18: 1)$, linoleic $(18: 2)$, linolenic $(18: 3)$, or arachidonic $(20: 4)$ acid was added to methanol solution containing either $\alpha$-tocopherol $(\bullet)$ or one of its model compounds $2(\boldsymbol{\Delta}), 3(\diamond)$, and $4(\boldsymbol{\square})$ at the concentration of $1.0 \times 10^{-4} \mathrm{M}$.

number of double bonds in the fatty acid. The decrements in fluorescence intensity are proportional to the concentration of the fatty acid, and the decrements for all the chromanols due to the addition of the fatty acid at a specified concentration are almost the same. Therefore, the isoprenoid side chain of $\alpha$-tocopherol is concluded to be unessential for complex formation. In addition, although the fluorescence of chromanols was quenched dependent on the number of double bonds in fatty acids, the quenching efficiency of arachidonic acid was nearly equal to that of linolenic 
acid (Fig. 2). Using the 1:1 mixture of arachidonic acid and linolenic acid, an additive, not synergistic, quenching effect was observed. There is no large difference between the $\alpha$-tocopherol-associating capabilities of arachidonic and linolenic acids. These results show that the chromanol moiety of $\alpha$-tocopherol is important for complex formation and more than three methylene-interrupted $\mathrm{Z}$ double bonds of a fatty acid for better interaction.

Previously, Erin et al. proposed that the interaction of $\alpha$-tocopherol with an unsaturated fatty acid is due to the hydrogen bonding between the hydroxy group on the chromanol moiety and a carboxyl group of fatty acids(4). We observed,

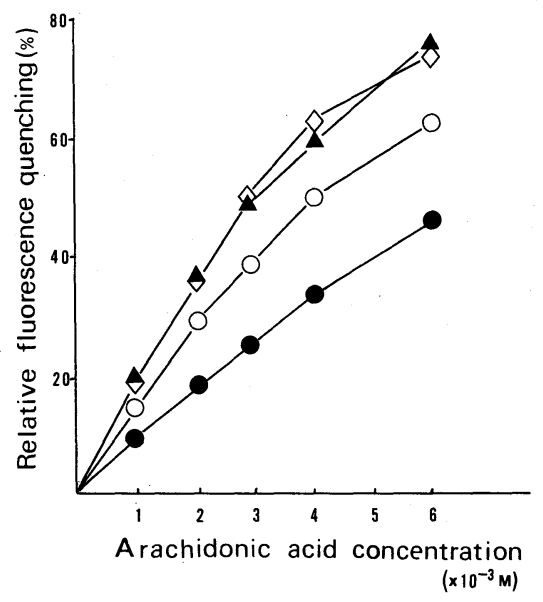

Fig. 3. Relative fluorescence quenching of $\alpha$-tocopherol and its derivatives in methanol by arachidonic acid concentration. $\alpha$-Tocopherol $(\bullet), \alpha$-tocopheryl acetate $(\boldsymbol{\Lambda})$, $\alpha$-tocopheryl methyl ether $(\bigcirc)$, or 6-deoxy- $\alpha$-tocopherol $(\diamond)$ was dissolved in methanol solutions of arachidonic acid. The intensity of fluorescence of $\alpha$-tocopherol is about twenty times as high as those of the derivatives in methanol.

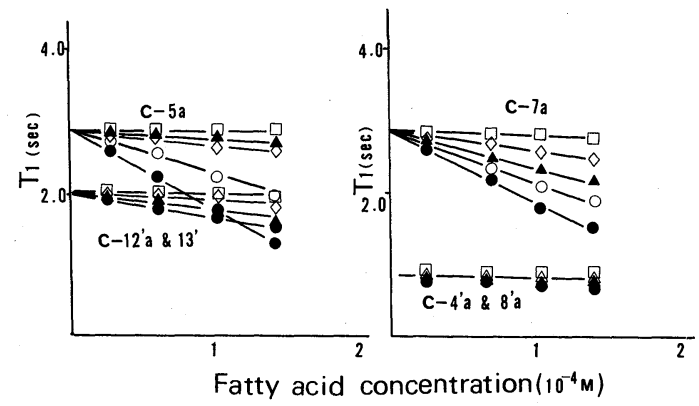

Fig. 4. Changes in the ${ }^{13} \mathrm{C}$ spin-lattice relaxation time $\left(\mathrm{T}_{1}\right)$ of $\alpha$-tocopherol by fatty acid concentration. Stearic $(\square)$, oleic $(\diamond)$, linoleic $(\Delta)$, linolenic $(O)$, or arachidonic (৫) acids was added to $1.2 \times 10^{-3} \mathrm{~N} \alpha$-tocopherol in methanol- $d_{4}$. At $20^{\circ} \mathrm{C}$, the ${ }^{13} \mathrm{C} \mathrm{T}_{1}$ relaxation times were measured. 
however, that when arachidonic acid was added to the methanol solution of $\alpha$ tocopheryl acetate, $\alpha$-tocopheryl methyl ether or 6-deoxy $\alpha$-tocopherol, the flurescence of these derivatives was much more quenched than that of $\alpha$-tocopherol itself (Fig. 3). Since each $\alpha$-tocopherol derivative bearing no hydroxy group can form a complex with a fatty acid more easily than $\alpha$-tocopherol, no involvement of the hydroxy group in $\alpha$-tocopherol in complex formation is deduced.

In order to clarify which carbon atoms in the $\alpha$-tocopherol molecule are involved in complex formation, we examined the ${ }^{13} \mathrm{C}$ spin-lattice relaxation time $\left(\mathrm{T}_{1}\right)$ of each carbon atom of $\alpha$-tocopherol in the complex by ${ }^{13} \mathrm{C}-\mathrm{NMR}$ spectroscopy. It is expected that as the atoms participate in the complex formation, their $T_{1}$ values will be reduced due to their restricted mobility. Figure 4 shows that when arachidonic acid was added to $\alpha$-tocopherol in methanol, the $T_{1}$ values of the 5aand $7 \mathrm{a}$-carbon atoms in the chromanol moiety were reduced to the greatest extent, and that those of the $12^{\prime} \mathrm{a}$ - and $13^{\prime}$-carbon atoms in the terminus of the isoprenoid side chain were also reduced to a small extent. Using a given concentration of the fatty acids, the $T_{1}$ values of the $5 \mathrm{a}$ - and $7 \mathrm{a}$-carbon atoms decreased with an increase in the number of double bonds in the fatty acids. No changes, however, in the $T_{1}$ values of the $4^{\prime} \mathrm{a}$ - and 8 'a-carbon atoms in the middle of the side chain were observed. Thus, methyl groups in the chromanol moiety seem to contribute to complex formation rather than those in the isoprenoid side chain. Consequently, it appears that due to an association of the methyl groups borne on the aromatic moiety of $\alpha$-tocopherol with the $Z$ double bonds of an unsaturated fatty acid, a complex is formed, and that for better interaction, more than three methyleneinterrupted $\mathrm{Z}$ double bonds of a fatty acid are necessary.

In the present study, the complex formation was observed in homogeneous solution. Thus, it is of great importance to know whether the above phenomena are observed in membrane systems. Our results are in good agreement with the finding that three methyl groups borne on the aromatic moiety of $\alpha$-tocopherol have a strong affinity toward unsaturated lipids in liposomes $(11,13)$. Hence, it seems likely that the interaction of $\alpha$-tocopherol with an unsaturated fatty acid in homogeneous solution is similar to that in membrane systems, although it is difficult at present to clarify the mechanism of the complex formation. On the basis of these results, our findings are incompatible with the hypothesis of Diplock and Lucy on the interaction of vitamin $\mathrm{E}$ with polyunsaturated lipids (1), making the validity of this hypothesis questionable. Further work is currently underway to investigate the interaction of $\alpha$-tocopherol with lipids in liposomes and biomembranes such as a membrane of erythrocytes.

This work was supported in part by Grants-in-Aid from the Ministry of Education, Science and Culture of Japan (No. 59570930), and Tokyo Metropolitan Institute of Gerontology. 


\section{REFERENCES}

1) Diplock, A. T., and Lucy, J. A. (1973): The biochemical modes of action of vitamin E and selenium: A hypothesis. FEBS Lett., 29, 205-210.

2) McCay, P. B., Fong, K. L., and King, M. M. (1978): Possible role of vitamin $E$ as a free radical scaveng and singlet oxygen quencher in biological systems which initiate radicalmediated reactions, in Tocopherol, Oxygen and Biomembranes, ed. by deDuve, C., and Hayaishi, O., North-Holland and Biomedical Press, Amsterdam, pp. 41-57.

3) Fukuzawa, K., Ikeno, H., Tokumura, A., and Tsukatani, H. (1979): Effect of $\alpha$ tocopherol incorporation on glucose permeability and phase transition of lecithin liposomes. Chem. Phys. Lipids, 23, 13-22.

4) Erin, A. N., Skrypin, V. V., and Kagan, V. E. (1985): Formation of $\alpha$-tocopherol complexes with fatty acids. Nature of complexes. Biochim. Biophys. Acta, 815, 209-214.

5) Maggio, B., Diplock, A. T., and Lucy, J. A. (1977): Interactions of tocopherols and ubiquinones with monolayers of phospholipids. Biochem. J., 161, 111-121.

6) Fukuzawa, K., Hayashi, K., and Suzuki, A. (1977): Effects of $\alpha$-tocopherol and analogues on lysosome membranes and fatty acid monolayers. Chem. Phys. Lipids, 18, 39-48.

7) Diplock, A. T., Lucy, J. A., Verrinder, M., and Zieleniewski, A. (1977): $\alpha$-Tocopherol and the permeability to glucose and chromate of unsaturated liposomes. FEBS Lett., 82, 341-344.

8) Srivastava, S., Phadeke, R. S., Govil, G., and Rao, C. N. R. (1983): Fluidity, permeability and antioxidant behavior of model membranes incorporated with $\alpha$ tocopherol and vitamin E acetate. Biochim. Biophys. Acta, 734, 353-362.

9) Cushley, R. J., and Forest, B. J. (1976): ${ }^{13} \mathrm{C}$ Fourier transform nuclear magnetic resonance. XII. Structure of the phytol-lecithin bilayer. Can. J. Chem., 54, 2059-2067.

10) Urano, S., and Matsuo, M. (1985): Synthesis of ${ }^{13} \mathrm{C}$-labeled vitamin $\mathrm{E}$ and interaction between vitamin $\mathrm{E}$ and phospholipid in liposomes, in Synthesis and Applications of Isotopically Labeled Compounds, ed. by Muccino, R. R., Elsevier, Amsterdam, pp. 517-518.

11) Urano, S., Iida, M., Otani, I., and Matsuo, M. (1987): Membrane stabilization of vitamin E: Interactions of $\alpha$-tocopherol with phospholipids in bilayer liposomes. Biochem. Biophys. Res. Commun., 146, 1413-1418.

12) Urano, S., Nakano, S., and Matsuo, M. (1983): Synthesis of $d l-\alpha$-tocopherol and $d l-\alpha-$ tocotrienol. Chem. Pharm. Bull., 31, 4341-4345.

13) Urano, S., Yano, K., and Matsuo, M. (1988): Membrane stabilizing effect of vitamin E: Effect of $\alpha$-tocopherol and its model compounds of fluidity of lecithin liposomes. Biochem. Biophys. Res. Commun., 150, 469-475. 